COMPUTER SCIENCE

\title{
NETWORK-CALCULUS-BASED ANALYSIS OF POWER MANAGEMENT IN VIDEO SENSOR NETWORKS
}

\author{
YANCHUAN CAO
}

\section{Thesis under the direction of Professor Yuan Xue}

This thesis considers two important issues for video sensor networks, (1) timely delivery of captured video stream and (2) energy-efficient network design. Based on network calculus, it presents a unified analytical framework that is able to quantitatively weigh the tradeoff between these two factors. In particular, it derives the service curve, buffer and delay bound under singlehop and multi-hop scenarios for different power management policies. To the best of our knowledge, this is the first work that extends the network calculus theory to the domain of wireless network with the consideration of power management and wireless interference. Our analysis has been validated through experiments conducted on a video sensor network testbed.

Approved

Date 


\title{
NETWORK-CALCULUS-BASED ANALYSIS OF POWER MANAGEMENT IN VIDEO SENSOR NETWORKS
}

\author{
By \\ Yanchuan Cao \\ Thesis \\ Submitted to the Faculty of the \\ Graduate School of Vanderbilt University \\ in partial fulfillment of the requirements \\ for the degree of \\ MASTER OF SCIENCE \\ in \\ Computer Science \\ December, 2008 \\ Nashville, Tennessee
}

Approved:

Professor Yuan Xue

Date

Professor Yi Cui

Date 
TABLE OF CONTENTS

Page

LIST OF FIGURES . . . . . . . . . . . . . . . . iii

Chapter

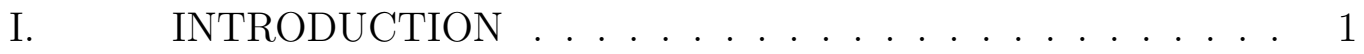

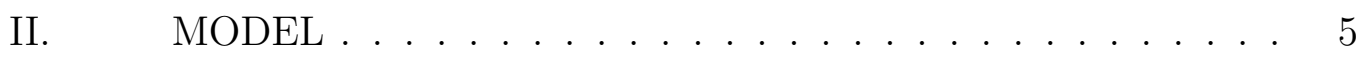

System Model . . . . . . . . . . . . . . . . . . 5

Traffic Model . . . . . . . . . . . . . . . . 7

Energy Model . . . . . . . . . . . . . . . . . . . . 9 9

III. ANALYSIS FOR SINGLE-HOP WIRELESS NETWORK . . 10

IV. ANALYSIS FOR MULTI-HOP WIRELESS NETWORK . . . 14

Contention Model . . . . . . . . . . . . . . . . . 14

Analysis within Single Clique . . . . . . . . . . . 16

Refined Bounds with Spacial Reuse . . . . . . . . . . . 17

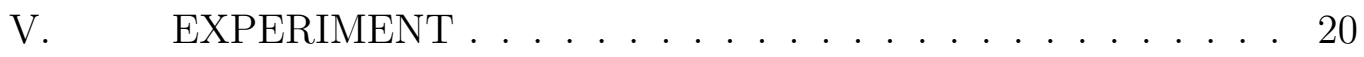

Experiment Setup . . . . . . . . . . . . . . . 20

Experiment Results . . . . . . . . . . . . . . . . . . . 22

VI. RELATED WORKS ................ . 33

VII. CONCLUSION .................. 35

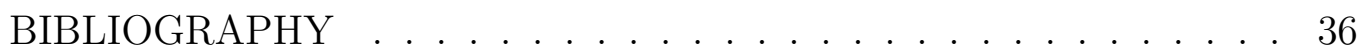




\section{LIST OF FIGURES}

Figure

Page

I.1. A Example explaining the correlation between application semantics and system resource condition. . . . . . . . . 2

II.1. Video Sensor Model. . . . . . . . . . . . . . . . . . . . . 5

II.2. Traffic Model. . . . . . . . . . . . . . . . . . . . . . . . 6

III.1. Single-Hop Scenario. . . . . . . . . . . . . . . . . . . . . . . 10

IV.1. Multi-Hop Scenario. . . . . . . . . . . . . . . . . . 14

V.1. Arrival Flow and Arrival Curve. . . . . . . . . . . . . 20

V.2. Departure Flow in Single-hop Scenario with 2 nodes. . . . . 21

V.3. Multi-hop Experiment Scenarios. . . . . . . . . . . . . 22

V.4. Departure Flow and Departure Curve in Single-hop Scenario (2 Nodes with Arrival Curve in Background). . . . . . . . . 23

V.5. Departure Flow and Departure Curve in Single-hop Scenario (4 Nodes with Arrival Curve in Background). . . . . . . . . 24

V.6. Delay and Delay Bound in Single-hop Scenario (2 Nodes) . . 25

V.7. Buffer and Buffer Bound in Single-hop Scenario (2 Nodes) . 26

V.8. Delay and Delay Bound in Multi-hop Scenario ( Scenario A in Fig. V.3 ) . . . . . . . . . . . . . . . . . . . . . . 27

V.9. Buffer and Buffer Bound in Multi-hop Scenario ( Scenario A in Fig. V.3 ) . . . . . . . . . . . . . . . . . . . 28 
V.10. Delay and Delay Bound in Multi-hop Scenario ( Scenario B in Fig. V.3 ) . . . . . . . . . . . . . . . . . . . . . . . . . . . . . . . 29

V.11. Buffer and Buffer Bound in Multi-hop Scenario ( Scenario B in Fig. V.3 ) . . . . . . . . . . . . . . . . . 30

V.12. Delay and Delay Bound in Multi-hop Scenario ( Scenario C in Fig. V.3 ) . . . . . . . . . . . . . . . . . . . . . . . . . 31

V.13. Buffer and Buffer Bound in Multi-hop Scenario ( Scenario $\mathrm{C}$ in Fig. V.3 ) . . . . . . . . . . . . . . . . . . . . . 32 


\section{CHAPTER I}

\section{INTRODUCTION}

The convergence of microelectronics and wireless communication leads to the emergence of wireless networks of sensor devices, which are capable of sensing, data processing and communication. Video sensor networks are equipped with video cameras as front end and are capable of providing video surveillance in many application scenarios, such as remote health care and traffic control. Many challenges arises in the design of video sensor network due to the conflict between its QoS requirement and the limited resource availability.

In this thesis, we consider two critical issues for video sensor network, (1) timely delivery of captured video stream and (2) energy-efficient network design. The first goal, driven by the application needs, e.g., video surveillance, targets to deliver the data acquired at a sensor node to the collection point in a timely fashion, or more strictly, within bounded delay. The second goal, driven by the resource scarcity of wireless nodes running on finite battery power, targets to maximize the network lifetime by carefully orchestrating the energy consumption of each node to the minimum degree and equitable manner.

Existing research on wireless (video) sensor networks usually considers one of the three interdependent aspects: delay $[11,19]$, bandwidth $[10,22,1]$ 


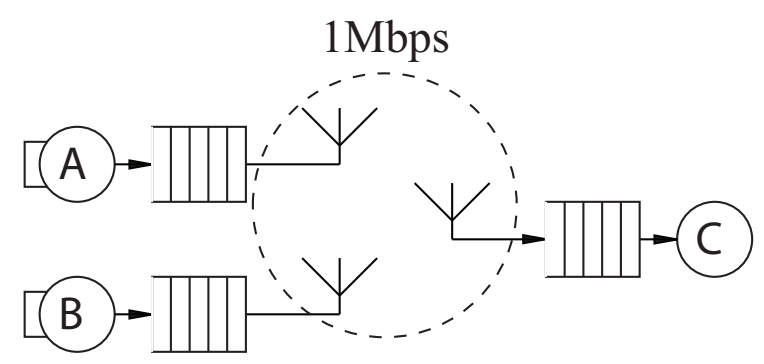

Figure I.1: A Example explaining the correlation between application semantics and system resource condition.

and lifetime $[6,9,8,12,23,5]$. Some works consider the tradeoff between two factors $[17,3,13]$. However, no theoretical framework exists in the existing literature that provides an analytical study of the tradeoff between bandwidth and delay requirement and energy consumption, especially under bursty traffic and power management.

Fig. I.1 illustrates a simple scenario to demonstrate the challenge. Among the three nodes shown in the picture, A and B are two video sensor nodes which can both directly connect to the data sink C. The achievable bandwidth of the shared wireless medium is $1 \mathrm{Mbps}$. Different solutions can be examined to explore the tradeoff between packet delay and power consumption. Enforcing power awareness in scheduling management, A and B alternatively transmit. When A is sleeping, B takes the entire 1Mbps bandwidth, and vice versa. Depending on the time quantum that $\mathrm{A}$ and $\mathrm{B}$ switch, large time quantum will reduce the switching overhead and save power accordingly at the cost of longer packet delay, while small time quantum will achieve the opposite. In the extreme case, with no power management, each node will 
always keep up and get itself 500Kbps bandwidth through contention, hence achieves the minimum packet delay.

Evidently, the goals of reducing packet delay and achieving energy efficiency are far from orthogonal, and actually contradictory from each other. This goal becomes even more challenging if we consider the bursty and nonuniform traffic in the video sensor networks. As such, we call for a unified framework that is able to quantitatively weigh the tradeoff of these two factors. Moreover, it should answer to the system objective (e.g., the packet delay bound) by setting the design parameter (e.g., maximum time quantum to meet the specified delay bound).

In light of such need, we propose an analytical framework to evaluate the tradeoff among video transmission requirements, buffer, delay and power consumption. Based on the previous results in network calculus [2], we investigate the service curve, buffer and delay bounds for both single-hop and multi-hop wireless network under power management. Such analytical results are important to guide the design of power management schedules that could minimize the energy consumption while providing bounded delay guarantee. Although considerably deriving from the previous results in min-plus network calculus, our work is justified by the following unique contributions: (1) This work is the first one that considers the effect of power management on the service curve and provides close-form analytical results for buffer and delay bounds; (2) This work extends the network calculus analysis to the domain of wireless network where flows not only contend in the temporal 
domain but also spatial domain, and provides the basis of interference-aware delay analysis for wireless networks. The rest of this thesis is organized as follows. We present the system model in Chapter II, the analysis of delay bound for single-hop wireless network in Chapter III and multi-hop network in Chapter IV. We evaluate our analysis in an experiment-based study in Chapter V, and conclude the thesis in Chapter VII. 


\section{CHAPTER II}

\section{MODEL}

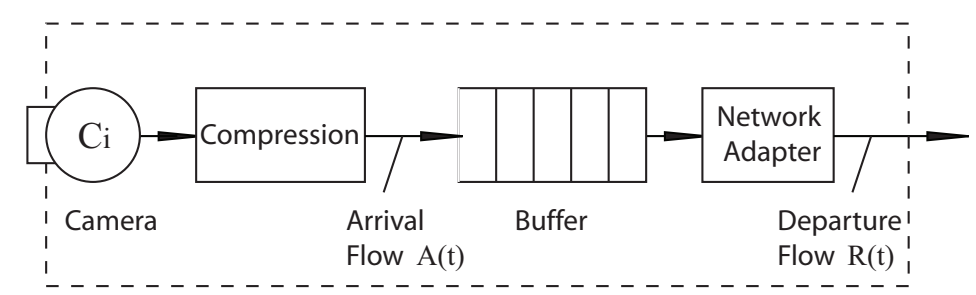

Figure II.1: Video Sensor Model.

\section{System Model}

We consider a wireless sensor network with a set of $N$ video sensors. Fig. II.1 illustrate the architecture of one sensor node. As shown in the figure, a video camera is used as the sensor front. Motion of objects in observed area will be captured as video images and compressed into a video stream. The compressed data abstract the difference between every adjacent raw video frames, recorded motion in raw data flow with more 'severe' object movement, in terms of more pixel difference, will cause larger amount of compressed data flow. The compressed video stream will be sent to a buffer for transmission.

In the following discussions, we call such compressed video stream arrival flow. In order to save energy, power management is adopted for the network 
adaptor, which periodically turns the adaptor into sleep. When awake, the network adaptor retrieves video packets from the buffer and transmit them in a FIFO fashion. The data stream transmitted from the network adaptor is called departure flow.

The video stream will be delivered from the video sensors to a data sink. In this thesis, we consider both single-hop networks and multi-hop networks. For simplicity, we consider the achievable network capacity as $C$.

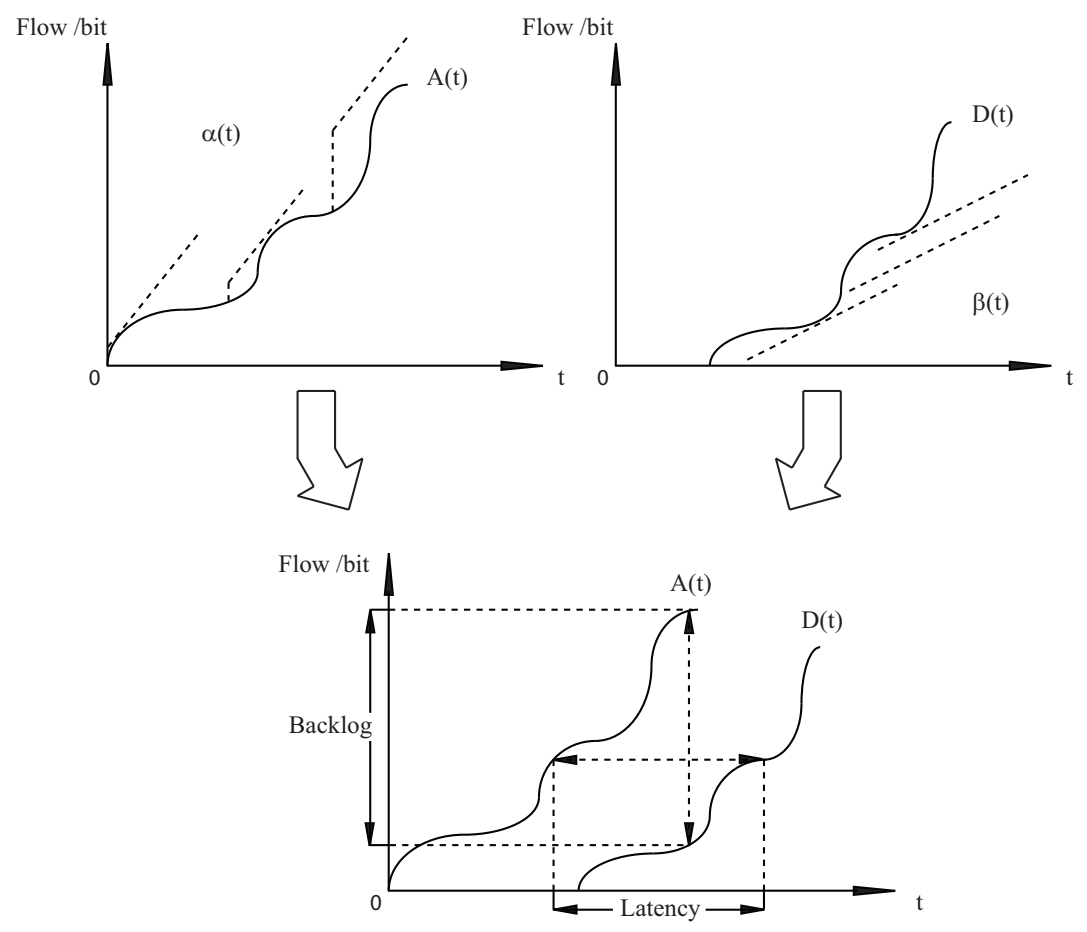

Figure II.2: Traffic Model.

\section{Traffic Model}


The occurrence of interested events in the surveillance area are random and bursty. which produces fluctuated traffic from arrival flows. To characterize such fluctuation, we model the arrival flow at node $i, i \in \mathcal{N}$ using its cumulative traffic $A_{i}(t)$, defined as the number of bits coming from the flow in time interval $[0, t]\left(A_{i}(0)=0\right)$, and $|\mathcal{N}|=N$. For a focused discussion on the impact of power management on delay, we adopt a fluid model for the arrival flows. Thus $A_{i}(t)$ is a continuous increasing function defined on continuous time domain. Further, we assume that the arrival flow traffic $A_{i}$ is constrained by a wide-sense increasing function $\alpha_{i}$, i.e.,

$$
A_{i}(t)-A_{i}(s) \leq \alpha_{i}(t-s) ; \forall s \leq t, t \geq 0
$$

$\alpha_{i}(t)$ is called the arrival curve of $A_{i}(t)$. In this thesis, we assume affine arrival curves for all video sensors, which is defined by $\alpha_{i}(t)=a_{i} \cdot t+b_{i}$. Having a affine arrival curve $\alpha_{i}(t)$ allows a source to send $b_{i}$ bits at once, but not more than $a_{i}$ bps over the long run, which means with a maximum flow rate of $a_{i}$. Fig. II.2 illustrates the relationship between the cumulative function $A(t)$ and the arrival curve $\alpha(t)$. Intuitively, for a randomly selected time period, the cumulative arrival function $A(t)$ always stays below the linear function defined by $\alpha_{i}(t)=a_{i} \cdot t+b_{i}$ within that time period.

Similarly, the departure flow from node $i$ could also be characterized by a cumulative function $D_{i}(t)$, which is defined as the traffic volume departed 
from node $i$ during time $[0, t]$. We further define service curve $\beta_{i}(t)$ of sensor node $i$ as a wide-sense increasing function, with $\beta(0)=0$ and

$$
D_{i}(t) \geq \inf _{s \leq t}\left(A_{i}(s)+\beta_{i}(t-s)\right)
$$

In network calculus [2], the arrival flow is denoted by $R(t)$ and the departure flow is denoted by $R^{*}(t)$.

When $\beta_{i}(t)$ is a continuous function, the use of infimum could be avoided. For continuous function $A_{i}(t)$ the above definition could be simplified as follows.

$$
D_{i}(t)-A_{i}(s) \geq \beta_{i}(t-s)
$$

The relation between arrival flow $A(t)$, departure flow $D(t)$, arrival curve $\alpha(t)$, and departure curve $\beta(t)$ are illustrated in Fig. II.2. For arrival flow $A(t)$, arrival curve $\alpha(t)$ serves as a piecewise upper bound for arrival flow rate; For departure flow $D(t)$, departure curve $\beta(t)$ serves as piecewise lower bound for service rate. Therefore, the vertical deviation between arrival flow $A(t)$ and departure flow $D(t)$ is the amount of backlogged flow data waiting to be transmitted, which should not exceed the buffer length of that sensor node. The horizontal deviation between arrival flow $A(t)$ and departure flow $D(t)$ is the latency that would be experienced by a bit of video flow, if all the data arrived before it is served before it. Intuitively, the buffer length, and 
delay are bounded by their corresponding deviation between arrival curve $\alpha(t)$ and departure curve $\beta(t)$.

\section{Energy Model}

In such a system, if assume Time division multiplex power management in single-hop scenario, the energy consumption will be contributed by three factors: data transmission, node sleeping, and slot switching. Denote the energy consumption rate of the three stages as $E_{t x}, E_{\text {sleep }}$, and $E_{\text {switch }}$, we have the Unit Energy Consumption,

$$
E_{\text {unit }}=\left(\frac{1}{N}\right) E_{t x}+\left(\frac{N-1}{N}\right) E_{\text {sleep }}+\left(\frac{1}{T}\right) E_{\text {switch }}
$$

Here unit refers to the energy consumption of each node during one time period $T$. For example, suppose $N$ nodes communicate with data sink in Time division multiplex fashion. Each node has a share of time $T_{i}$, and a whole round takes time $T$. For node $i$, during its transmission time slot, energy will be consumed with rate $E_{t x}$, and with rate $E_{\text {sleep }}$ for the rest of the period $T$. For round-robin, each node will have $\frac{1}{N}$ share of the period, transmitting, and $1-\frac{1}{N}$ share of the period, sleep. Between the state change of transmission and sleep, energy consumption rate will be $E_{\text {switch }}$. The switching happens once every period for each node. 


\section{CHAPTER III}

\section{ANALYSIS FOR SINGLE-HOP WIRELESS NETWORK}

We start with the simplest case of video sensor network - single-hop wireless network.

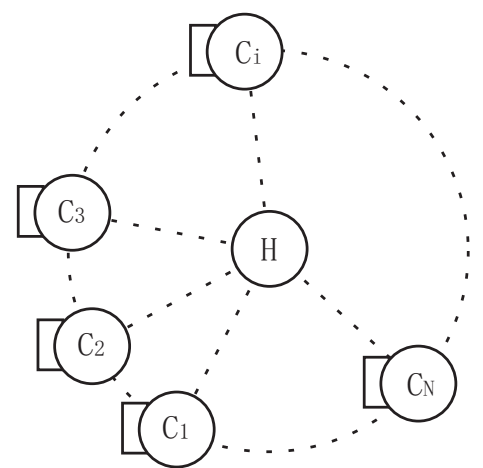

Figure III.1: Single-Hop Scenario.

In this case all sensor nodes could communicate with the data sink directly. These nodes are sharing the wireless channel capacity together. We assume that the power management are in a time-division fashion with pe$\operatorname{riod} T$. Each node $i$ will wakes up during time slot $T_{i}$ and transmit. All the other nodes will remain asleep until its turn to transmit. The above TDM scheduling serve as a power management scheme can as well reduce the wireless contention. 
Now we proceed to analyze the delay experienced by the data flow from node $i$. First we study the service curve $\beta_{i}(t)$ of node $i$ under the above power management scheme. Recall that $C$ is the achievable capacity of the wireless network. Since only one node wakes up and transmit at a time, node $i$ is able to transmit at rate $C$ during its wake-up period, which leads to an average transmission rate of $\frac{C \cdot T_{i}}{T}$. Also node $i$ will wait at most $T-T_{i}$ for its next turn. Thus we have the following results for service curve at node $i$. All the proofs in this thesis are provided in our technical report [4] due to the space constraint.

Lemma 1 (Service Bound in Single-Hop Network) The service curve $\beta_{i}$ for node $i$ under time-division round-robin power management with wakeup time $T_{i}$ out of period $T$ is given by the following rate-latency function with rate $\frac{C \cdot T_{i}}{T}$ and latency $T-T_{i}$.

$$
\beta_{i}(\tau)=\frac{C \cdot T_{i}}{T}\left[\tau-\left(T-T_{i}\right)\right]^{+}
$$

At time $t$, the amount of data await in the buffer of node $i$ is called backlog $B_{i}$, which can be considered as the difference between the cumulative function of arrival flow and departure flow $A_{i}(t)-D_{i}(t)$. Recall that the cumulative function of the arrival flow $A_{i}(t)$ is $\alpha-$ smooth, with $\alpha$ as its arrival curve. Then the backlog at time $t$ satisfies 


\section{Lemma 2 (Buffer Bound in Single-Hop Network)}

$$
B_{i} \leq \sup _{s \geq 0}\{\alpha(s)-\beta(s)\}
$$

Such a backlog introduces a delay for the data stream. Formally we define virtual delay at time $t$ as

$$
d(t)=\inf _{\tau \geq 0}\{A(t) \leq D(t+\tau)\}
$$

which is the delay that would be experienced by a bit arriving at $t$ if all bits are received before it are served before it.

Now we describe the latency at time $t$ with the virtual delay $d(t)$.

Theorem 3 (Delay Bound for Single-hop Network) In a single-hop wireless sensor network under time-division round-robin power management, the delay for flow

$$
d(t)=\inf \{\tau \geq 0: A(t) \leq D(t+\tau)\}
$$

The definition of $d(t)$ is the delay that would be experienced by a bit arriving at time $t$ if all bits arrived before it are served before it. The intuition behind $d(t)$ is like this(See Fig. II.2), because both arrival and departure cumulative function $A(t)$ and $D(t)$ are wide-sense increasing, virtual delay $d(t)$ can be considered as shift $D(t)$ leftward, to the time point, at which $D(t)$ 
has the same value with $A(t)$, and $d(t)$ is the smallest value that $D(t)$ need to shift, which means departure flow $D(t)$ will cumulate to the same amount of bits as the arrival flow after a latency with the length of at least $d(t)$. We considered delayed bound instead of other metrics as jitter explicitly. Because if every packets in departure flow satisfy the delay bound, we may smooth out jitters at receiver side buffer with corresponding playback delay. 


\section{CHAPTER IV}

\section{ANALYSIS FOR MULTI-HOP WIRELESS NETWORK}

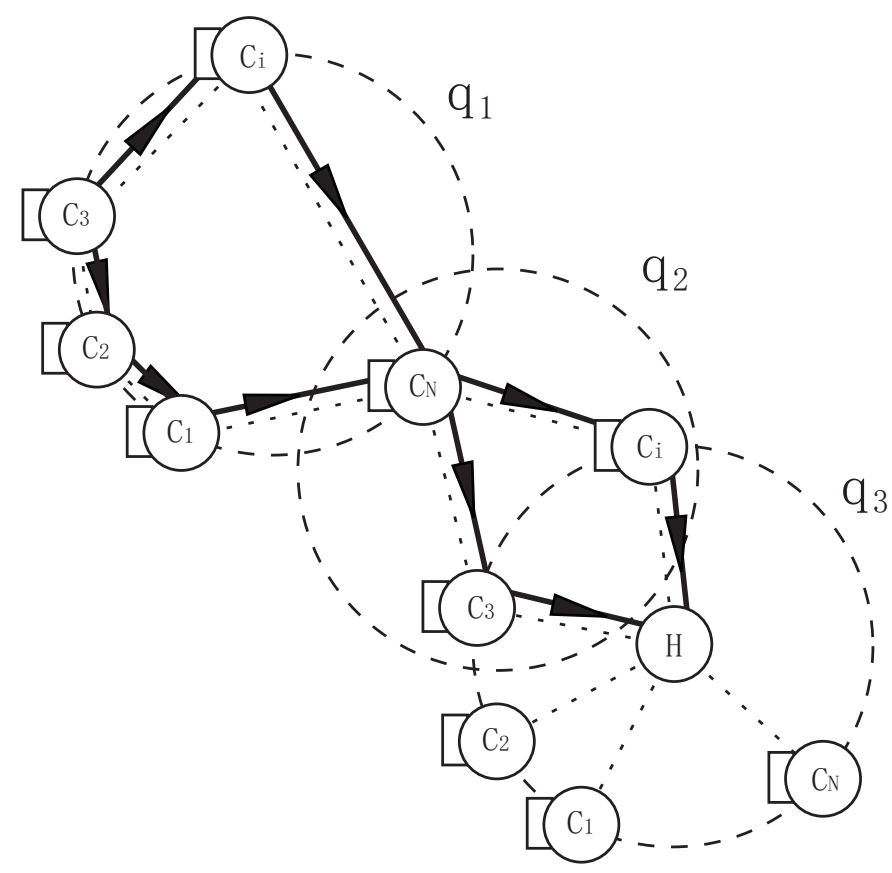

Figure IV.1: Multi-Hop Scenario.

\section{Contention Model}

A wireless video sensor network can be modelled as a graph $G=(\mathcal{N}, E)$. $E \subseteq 2^{\mathcal{N}}$ denotes the set of wireless links, which are formed by sensor nodes 
that are within the transmission range of each other. A wireless link $e \in E$ is represented by its end nodes $i$ and $j$, i.e., $e=\{i, j\}$

In such a network, there exists a set of end-to-end flows, denoted as $F$. Each flow $f \in F$ goes through single or multiple hops in the network, passing a set of wireless links $E(f)$. A single-hop data transmission in the flow $f$ along a particular wireless link is referred to as a subflow of $f$. We use the notation $S(S \subseteq E)$ to represent a set of wireless links in $G$, such that each of the wireless links in $S$ carries at least one subflow, i.e. the wireless link is currently not idle.

Based on the protocol model, flows in a wireless video sensor network contend for shared resources in a location-dependent manner: two subflows contend with each other if either the source or destination of one subflow is within the range of the source or destination of the other. Among a set of mutually contending subflows, only one of them may transmit at any given time. Formally, we consider a wireless contention graph $G_{c}=\left(V_{c}, E_{c}\right)$, in which vertices correspond to the wireless $\operatorname{links}\left(i . e ., V_{c}=S\right)$, and there exists an edge between two vertices if the subflows along these two wireless links contend with each other.

In a graph, a complete subgraph is referred to as a clique that is not contained in any other cliques [20]. In a wireless contention graph, the vertices in maximal clique represent a maximal set of mutually contending wireless links, along which at most one subflow may transmit at any given time. Denote the set of all maximal clique in $G_{c}$ as $Q$, and the achievable channel 
capacity(or bandwidth) at a clique $q \in Q$ as $C_{q}$. Each flow $f$ may pass through one or more maximal cliques, within each maximal clique $q$, sublinks of flow $f$, denoted as $S_{f}\left(S_{f}=V(q) \cap E(f)\right)$ will share the achievable channel bandwidth of clique $q$, in a time division multiplex manner. Each

sublink will have $\frac{C_{q}}{S_{f}}$ bps bandwidth, which is similar to the single-hop scenario. Sublinks of flow $f$ have to wait for its turns to transmit within each cliques , meanwhile, communication may be carry on simultaneously among the concatenating maximal cliques of flow $f$. Between every adjacent cliques delays will be cumulated, the concatenated service curve and bounds will be affected accordingly.

\section{Analysis within Single Clique}

We first study the service curve, buffer and delay bound within a single clique.

Lemma 4 (Service Curve in a Single Clique) The service curve $\beta_{q}$ for flow $f$, passing through maximal clique $q$ under time-division round-robin power management with number of sunlinks in clique $\left|S_{f}\right|=|V(q) \cap E(f)|$, is given by the following rate-latency function with rate $\frac{C_{q}}{\left|S_{f}\right|}$ and maximum possible latency $\left|S_{f}\right|\left(T-T_{i}\right)$.

$$
\beta_{q}(\tau)=\frac{C \cdot T_{i}}{T}\left[\tau-\left|S_{f}\right|\left(T-T_{i}\right)\right]^{+}
$$




\section{Lemma 5 (Buffer Bound in a Single Clique)}

$$
B_{q}=A_{q}(t)-D_{q}(t) \leq \sup _{s \geq 0}\left\{\alpha(s)-\beta_{q}(s)\right\}
$$

in which arrival curve $\alpha(s)$ remain the same, and service curve $\beta_{q}(s)=$ $\frac{C \cdot T_{i}}{T}\left[s-\left|S_{f}\right|\left(T-T_{i}\right)\right]^{+}$

Theorem 6 (Delay Bound in a Single Clique) In a multi-hop wireless sensor network with each maximal clique under time-division round-robin power management, the delay for flow $f$ within each clique $q$

$$
d(t)=\inf \left\{\tau \geq 0: D_{q-1}(t) \leq D_{q}(t+\tau)\right\}
$$

Lemma 4.1, Lemma 4.2, and Theorem 4.3 are based on analysis within each maximal clique. To get the flow-based service, buffer and delay bounds, we need to concatenate every pairs of adjacent cliques that flow $f$ pass through, and consider the accumulated constraint along the flow. Theorem 3.3 involves spacial reuse and concatenation at the same time, which is hard to resolve. To get more precise bounds, we divide the situation into two folds and consider them each at a time.

\section{Refined Bounds with Spacial Reuse}

If omit the affect of spacial reuse within each clique, and consider a treestyled hierarchical concatenation, in which, every flow are originated from 
leave nodes, and no data will be generated along each flow from leaf node(the camera) to root node(the end host). In this case, the concatenated result may be derived according to min-plus Algebra.

In min-plus Algebra $(\mathbb{R} \cup\{+\infty\}, \wedge,+)$, where the 'addition' is $\wedge$ and the 'multiplication' is + . an 'integral' of function $f$ becomes therefore

$$
\int_{0}^{t} f(s) d s=\inf _{0 \leq s \leq t}\{f(s)\}
$$

the convolution of two functions $\beta_{1}$ and $\beta_{2}$ is the function

$$
\left(\beta_{1} \otimes \beta_{2}\right)(t)=\inf _{0 \leq s \leq t}\left\{\beta_{1}(t-s)+\beta_{2}(s)\right\}
$$

Theorem 7 Assume a flow traverse two systems(nodes or cliques) $\mathcal{S}_{1}$ and $\mathcal{S}_{2}$ in sequence. If $\mathcal{S}_{i}$ offers a service curve of $\beta_{i}, i=1,2$ to the flow. Then the concatenation of the system offers a service curve of $\beta_{1} \otimes \beta_{2}$ to the flow, which is the min-plus convolution of the two service curves.

From Theorem \%, we can intuitively derive

Lemma 8 (Service Bound in Multi-Hop Network) Assume a flow traverse $N$ systems $\mathcal{S}_{i}, i=1,2, \ldots, N$ in sequence. If $\mathcal{S}_{i}$ offers a service curve of $\beta_{i}, i=1,2, \ldots, N$ to the flow. Then the concatenation of the system offers a service curve of $\prod_{i=1}^{N} \otimes \beta_{i}=\beta_{1} \otimes \beta_{2} \otimes \ldots \otimes \beta_{N}$ to the flow, which is the iterated min-plus convolution of the $N$ service curves. 
Therefore, we have the concatenated departure flow from system $\mathcal{S}_{N}$ as

$$
D_{N}=D_{0} \prod_{i=1}^{N} \otimes \beta_{i}=A_{1} \prod_{i=1}^{N} \otimes \beta_{i}
$$

With the service curve, we may get the buffer bound:

\section{Lemma 9 (Buffer Bound in Multi-Hop Network)}

$$
B_{q}=A_{q}(t)-D_{q}(t) \leq \sup _{s \geq 0}\left\{\alpha(s)-\beta_{q}(s)\right\}
$$

in which arrival curve $\alpha(s)$ remain the same, and service curve $\beta_{q}(s)=$ $\prod_{i=1}^{N} \otimes \beta_{i}$

For tree-like hierarchical concatenation we have

Theorem 10 (Delay Bound in Multi-Hop Network) Assume a flow traverse tree-like hierarchical systems $\mathcal{S}_{n, j}$ in sequence. If $\mathcal{S}_{n, j}$ offers a service curve of $\beta_{n, j}$ to the flow. Then the concatenation of the system offers a service curve of $\lambda[\cdot]$ to the flow, which is

$$
\left.\lambda[\bullet]=\sum_{j_{n-1}=1}^{N_{n-1}}\left[\sum_{j_{n-2}=1}^{N_{n-2}}\left[\ldots\left[\sum_{j_{0}=1}^{N_{0}} \bullet\right] \otimes \beta_{1, j_{1}}\right] \otimes \ldots\right] \otimes \beta_{n-1, j_{n-1}}\right] \otimes \beta_{n, j_{n}}
$$




\section{CHAPTER V}

\section{EXPERIMENT}

\section{$\underline{\text { Experiment Setup }}$}

The experiment was carried out on wireless video sensor platform. We use Stargate wireless testbed(XScale PXA255) as sensor node, with AmbiCom WL1100C-CF (802.11b) wireless network interface. The video sensor is Logitech Pro4000 camera, with USB connection, which connects to Stargate.

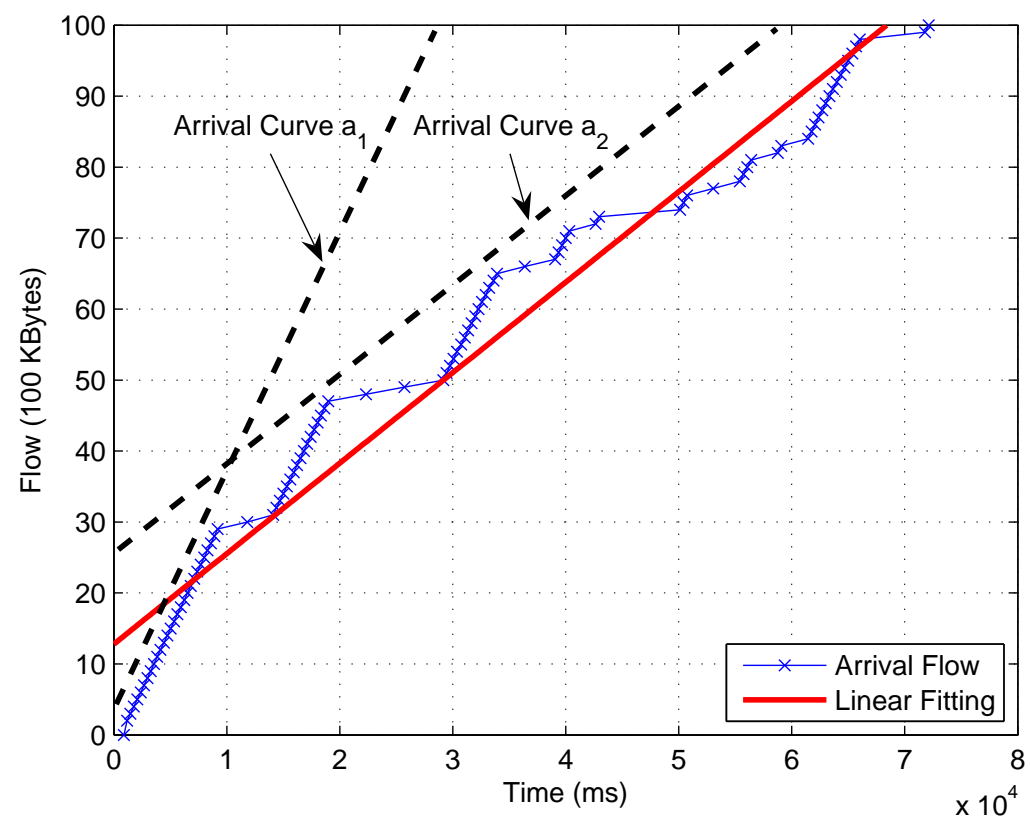

Figure V.1: Arrival Flow and Arrival Curve. 


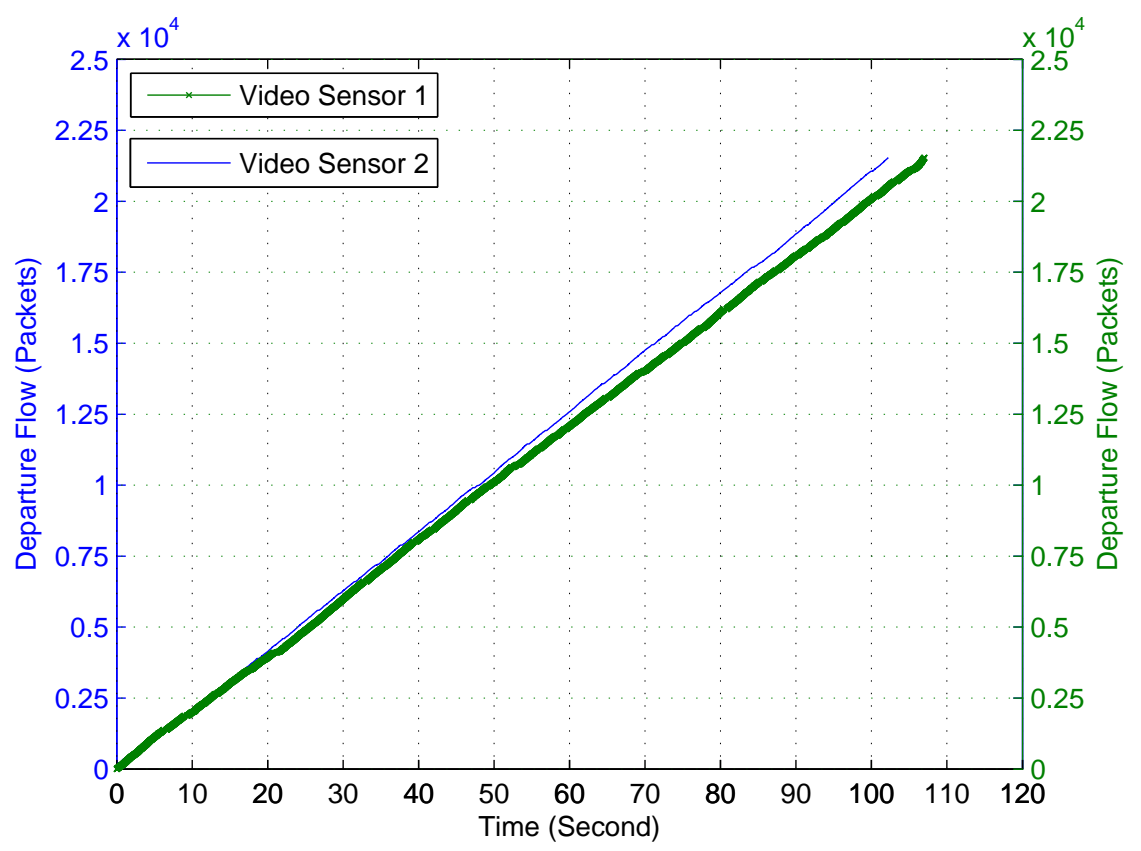

Figure V.2: Departure Flow in Single-hop Scenario with 2 nodes.

The video stream is generated by a modified video capture application vidcat on Stargate. The captured video stream will serve as Arrival Flow (See Fig. V.1) in our experiment. The Arrival Curve can be inferred with the maximum arrival flow rate. We generated the trace of Departure Flow (See Fig. V.2) by logging packets received at video sensor nodes and data sink (Fedora FC6 Laptop).

We tested our system with single-hop and multi-hop scenarios. In singlehop case, two or four video sensor nodes constantly send out video streams and contending for the wireless channel. The multi-hop experiment is carried out with three different routing configurations as showing in Fig. V.3. Video 


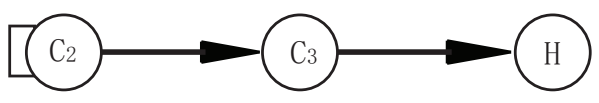

Scenario A

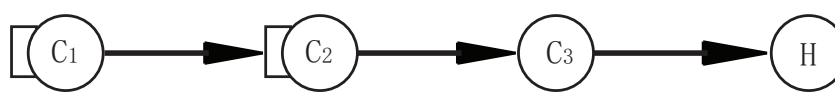

Scenario B

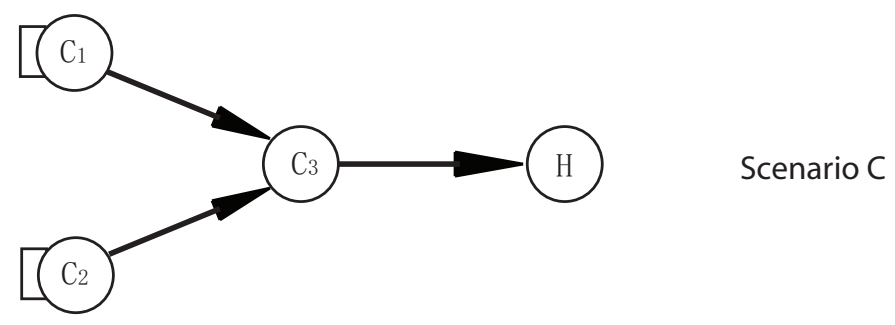

Figure V.3: Multi-hop Experiment Scenarios.

sensor node 1 and 2 will capture and transmit video streams, Video sensor node 3 serves as a dedicated router to data sink $H$, and does not capture video. In scenario A, only node 2 and 3 are presented and form a two-hop network to data sink. In scenario B, node 2 will send its own data as well as data forwarded for node 1 to data sink though node 3 . In scenario $\mathrm{C}$, node 1 and node 2 send video streams to data sink through contending for node 3 .

\section{Experiment Results}

With two nodes in single-hop scenario (See Fig. V.4), the departure curve will surpass the arrival curve, which means the transmission ability may well handle all the arrival flow and no data will be backlogged. With four nodes in single-hop scenario (See Fig. V.5), arrival flow will be delayed (See Fig. V.6) 


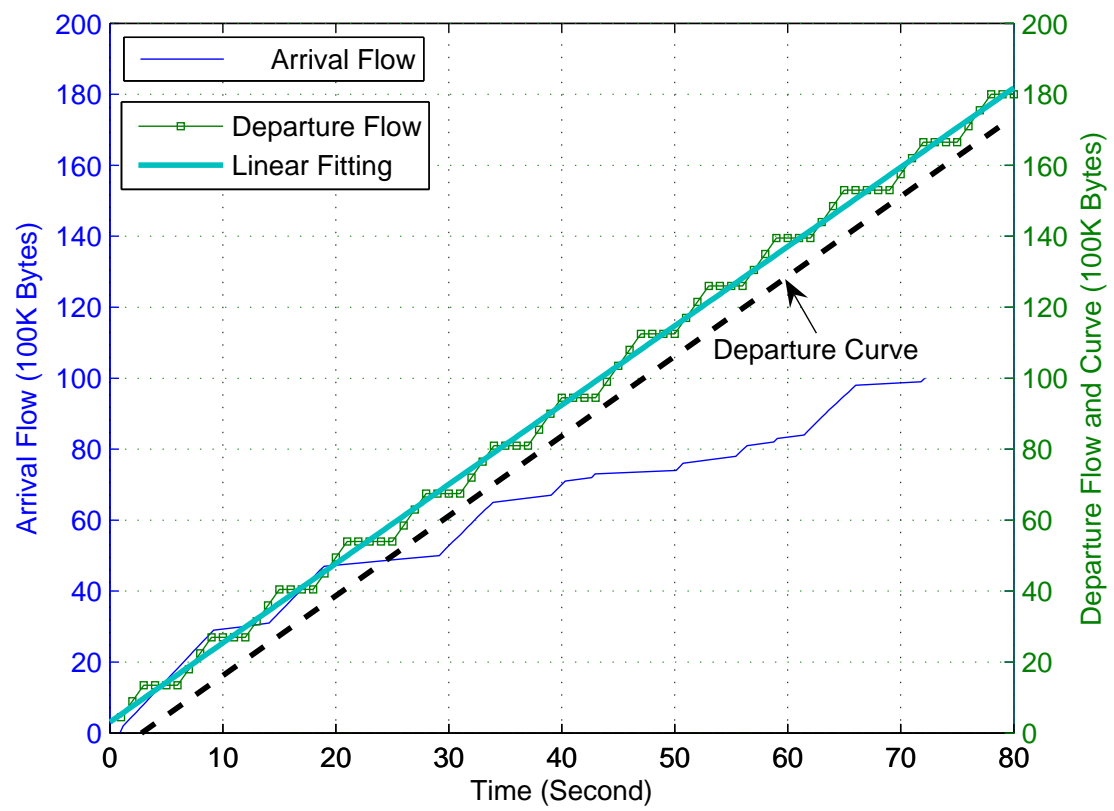

Figure V.4: Departure Flow and Departure Curve in Single-hop Scenario (2 Nodes with Arrival Curve in Background).

and backlogged (See Fig. V.7), but within the threshold of Delay Bound and Buffer Bound.

By logging the packet departure time at video sensor node and arrival time at data sink, we also measured the end-to-end delay and backlog length in aforementioned scenarios. In Fig. V.6, we get the delay of two singlehop nodes by measuring the horizontal deviation between arrival flow and departure flow in Fig. V.5. Similarly, in Fig. V.7, we get their backlog by measuring the vertical deviation between arrival flow and departure flow in Fig. V.5. 


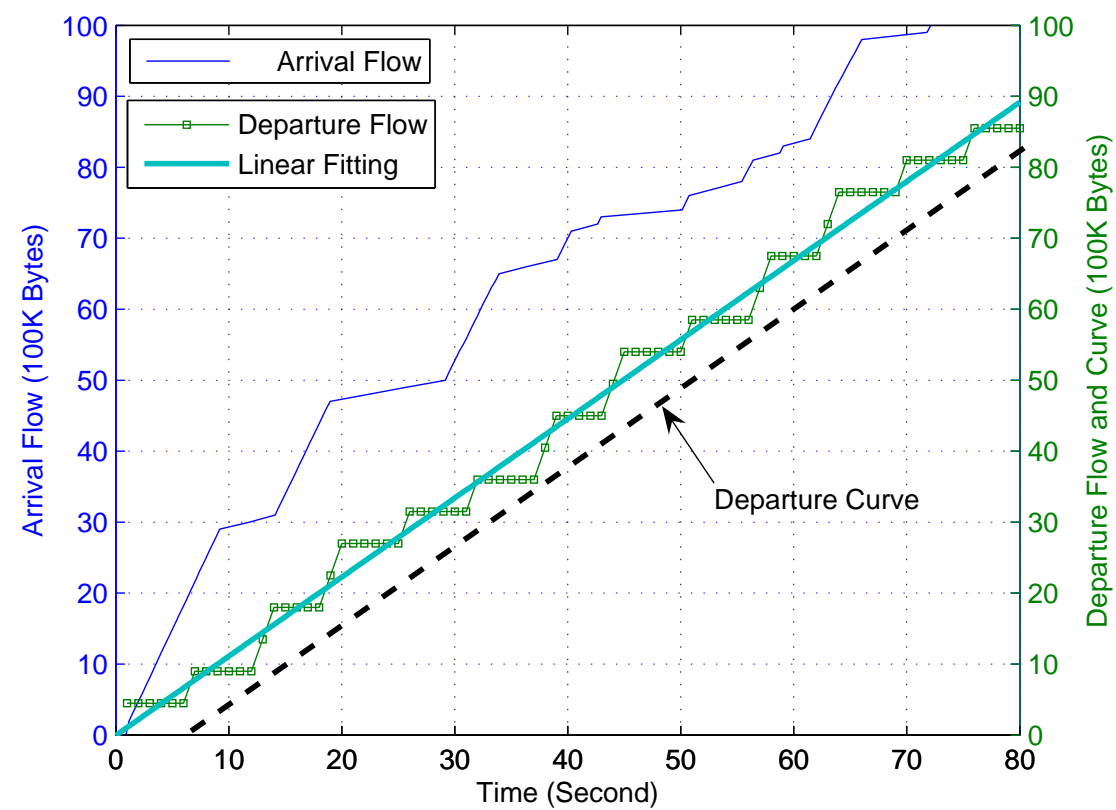

Figure V.5: Departure Flow and Departure Curve in Single-hop Scenario (4 Nodes with Arrival Curve in Background).

Similar experiment have also been performed with other multi-hop testbed setup: scenario A (See Fig. V.8 and Fig. V.9), scenario B (See Fig. V.10 and Fig. V.11), and scenario C (See Fig. V.12 and Fig. V.13).

From experiment result of scenario A (Fig. V.8 and Fig. V.9), we observed that, during the entire 300 seconds experiment, the longest delay caused by burst of event is around 6 second, and most of the time, the delay is around its average 2 second. For backlogged data, the largest backlog is about 6 frames $(1$ frame $=225 \mathrm{~K}$ Bytes $)$ with an average of between 1 and 2 frames. Therefore, both delay and backlog are bounded. If compare Fig. V.8 and Fig. V.9, we may find that when bursty event occur, the delay become longer and 


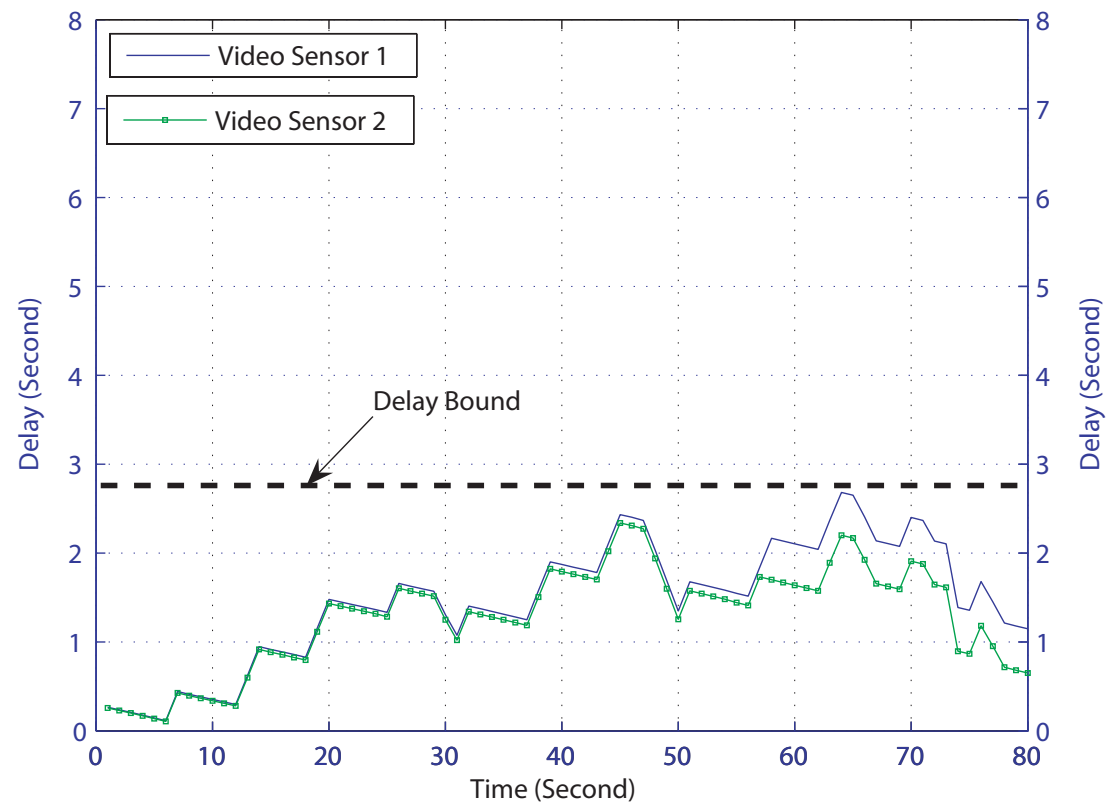

Figure V.6: Delay and Delay Bound in Single-hop Scenario (2 Nodes)

backlog get larger correspondingly. When transmission rate change rapidly, the delay become jitter, and backlog change get more intensively.

From experiment results of scenario B (Fig. V.10 and Fig. V.11), we may find similar phenomenon as observed in results of scenario A, besides, at the connection establishing stage, both node 1 and 2 shows jitter in delay and backlog, and get stable with the average around 4 seconds. If we compare the delay traces of node 1 and 2, we may find node 2's delay curve has the similar but larger shape with node 1 , and shifted right a little bit, since node 2 has to forward data from node 1 beside its own. The node with longer delay has larger backlog as well. 


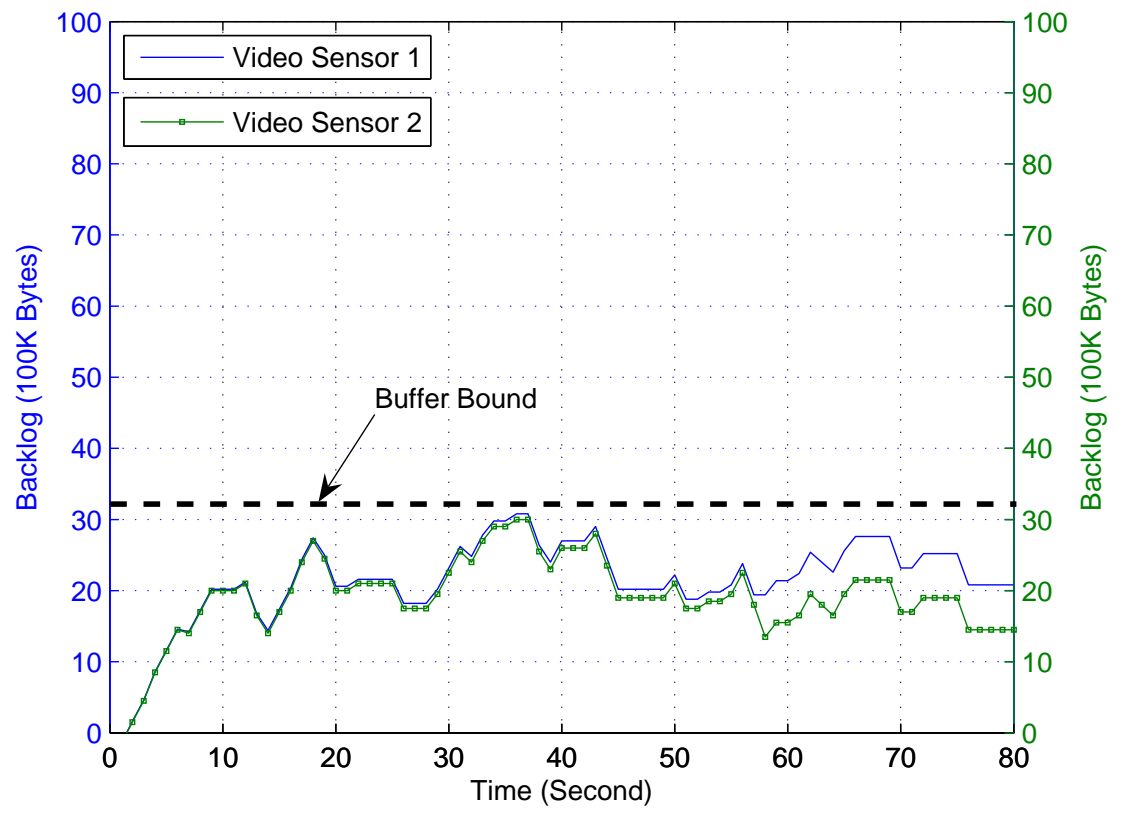

Figure V.7: Buffer and Buffer Bound in Single-hop Scenario (2 Nodes)

For results from scenario C (Fig. V.12 and Fig. V.13), delay curve from node 1 and 2 tend to be phase inversion of each other, and center with expectation value approximately, since both of them contend for node 3 . The average delay of the two nodes is around 3 seconds, which is "one hop" more efficient than scenario B on average. Senario C shows a more balanced load assignment than scenario B, in terms of delay and backlog, which hence means more evenly distributed energy consumption, and a longer overall battery life. 


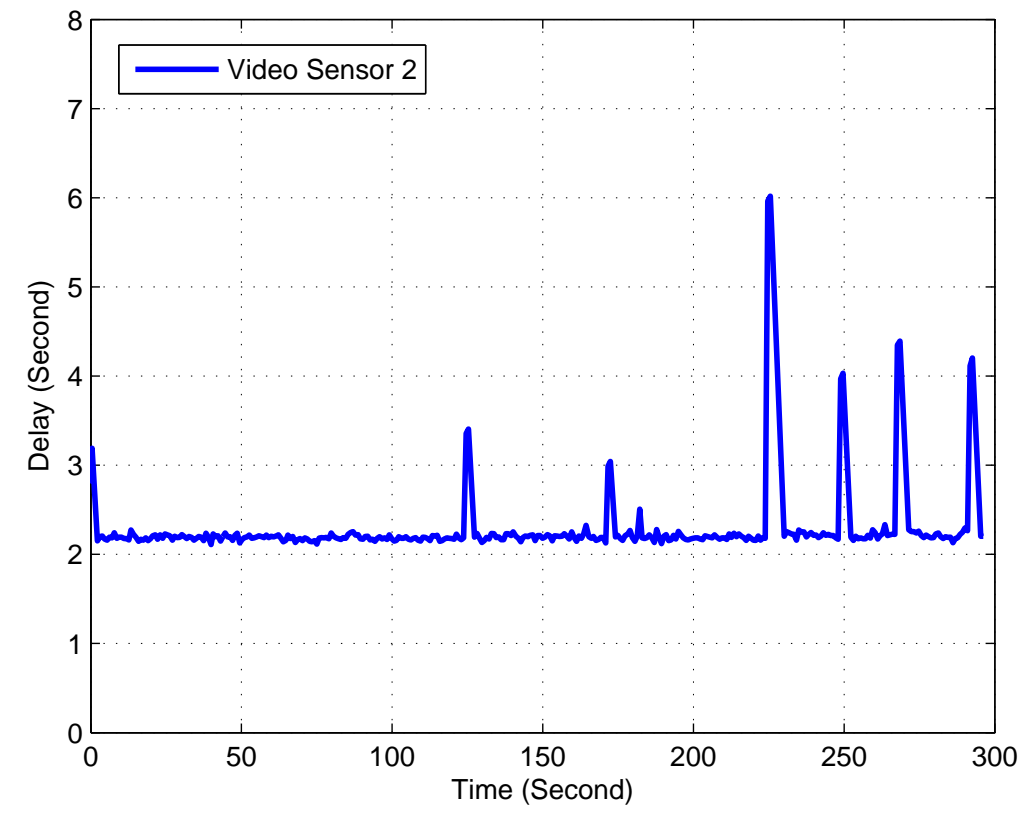

Figure V.8: Delay and Delay Bound in Multi-hop Scenario ( Scenario A in Fig. V.3 ) 


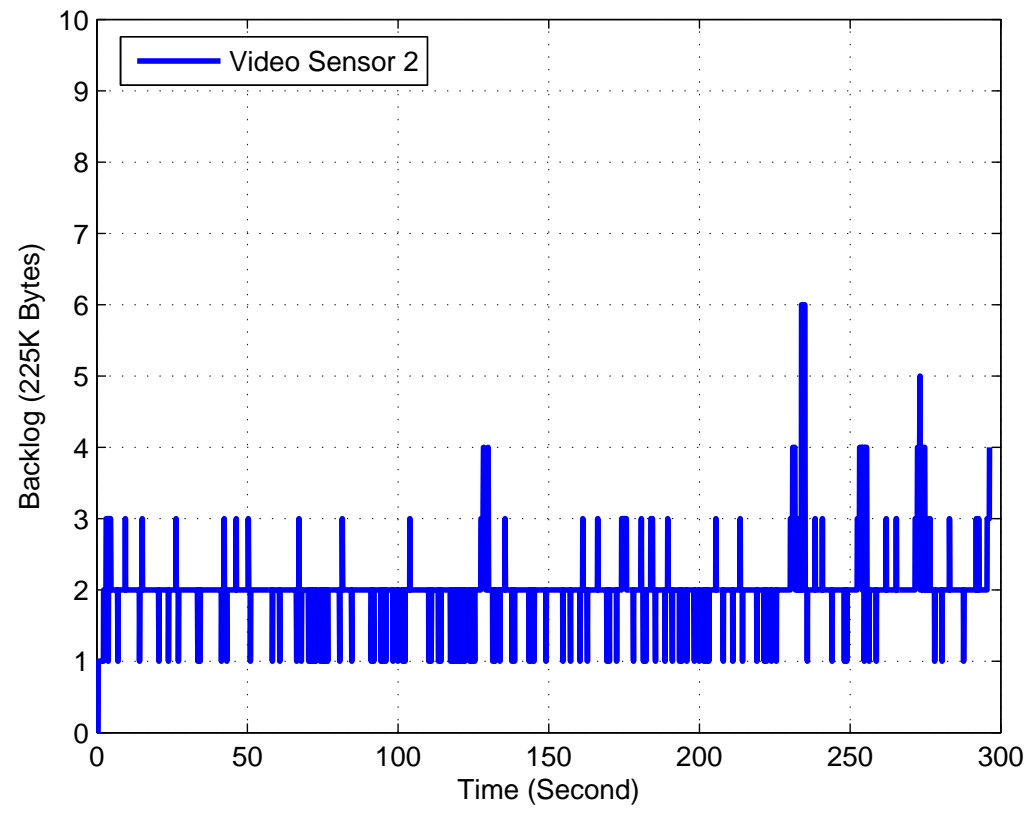

Figure V.9: Buffer and Buffer Bound in Multi-hop Scenario ( Scenario A in Fig. V.3 ) 


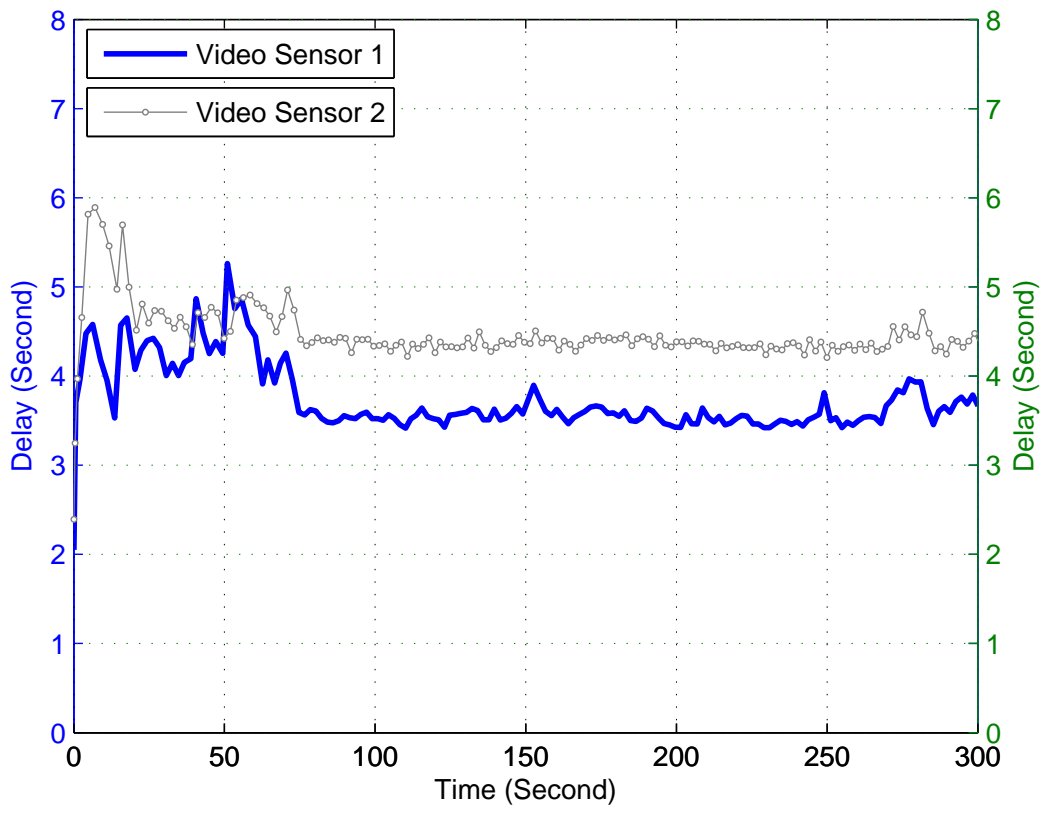

Figure V.10: Delay and Delay Bound in Multi-hop Scenario ( Scenario B in Fig. V.3 ) 


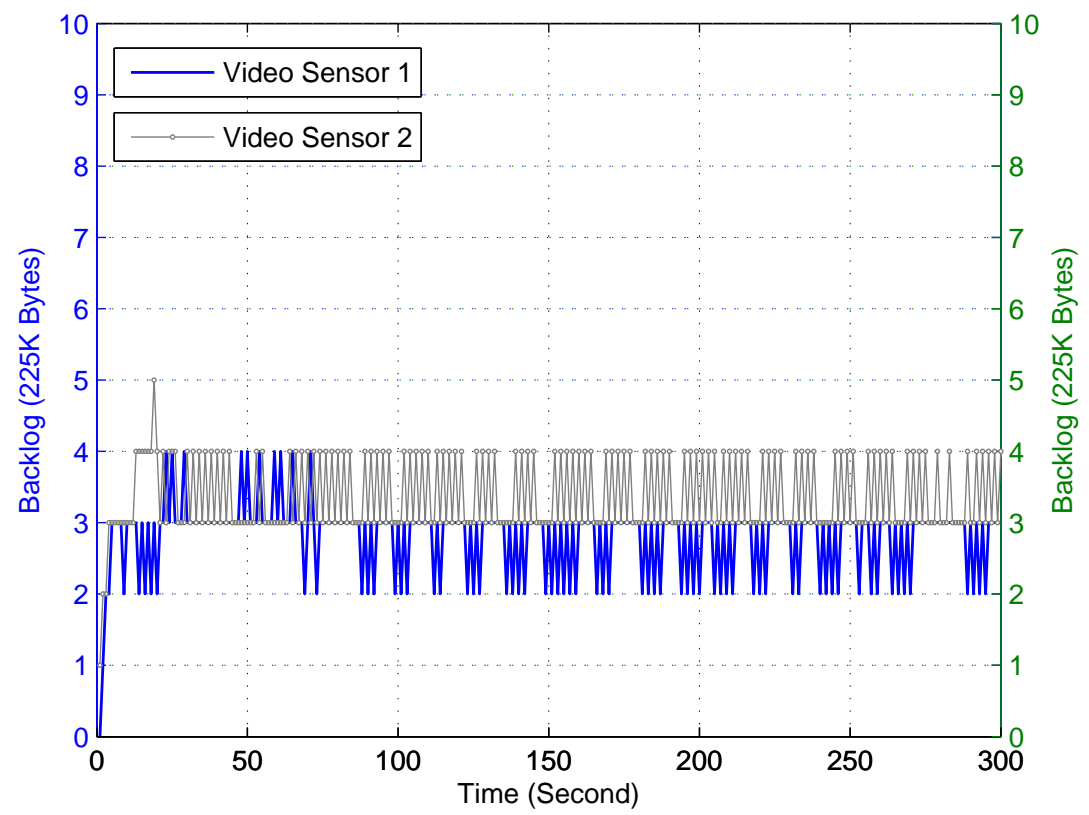

Figure V.11: Buffer and Buffer Bound in Multi-hop Scenario ( Scenario B in Fig. V.3 ) 


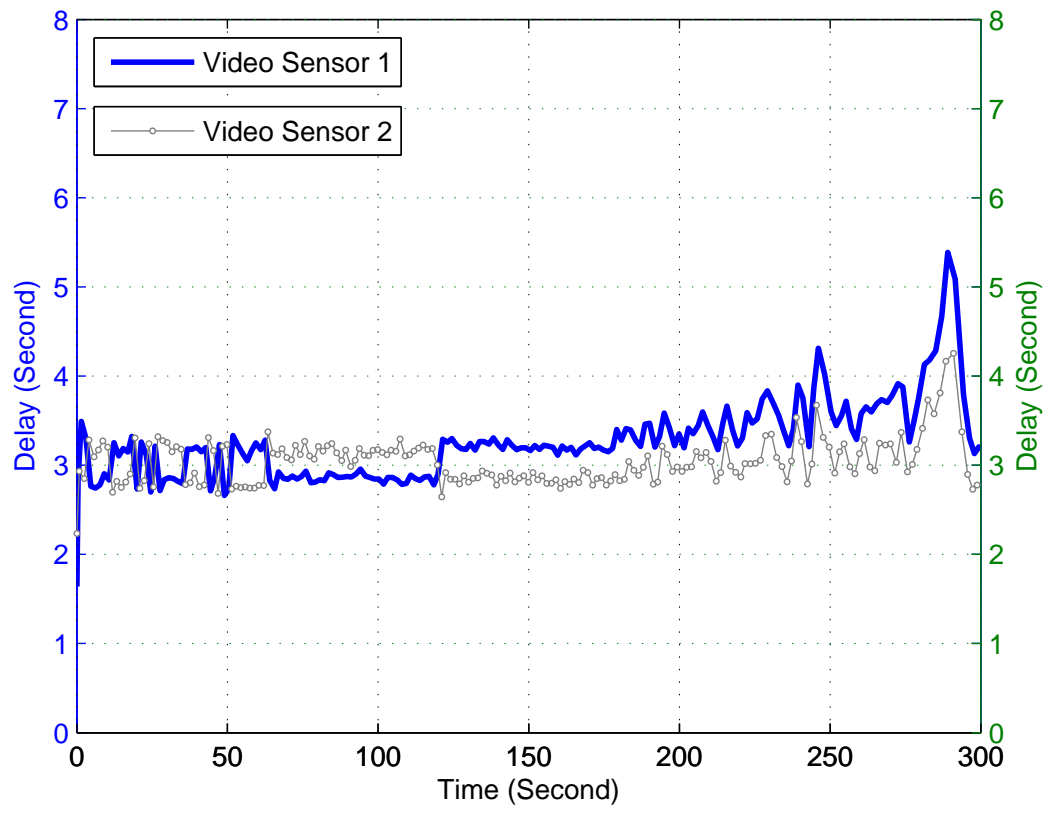

Figure V.12: Delay and Delay Bound in Multi-hop Scenario ( Scenario C in Fig. V.3 ) 


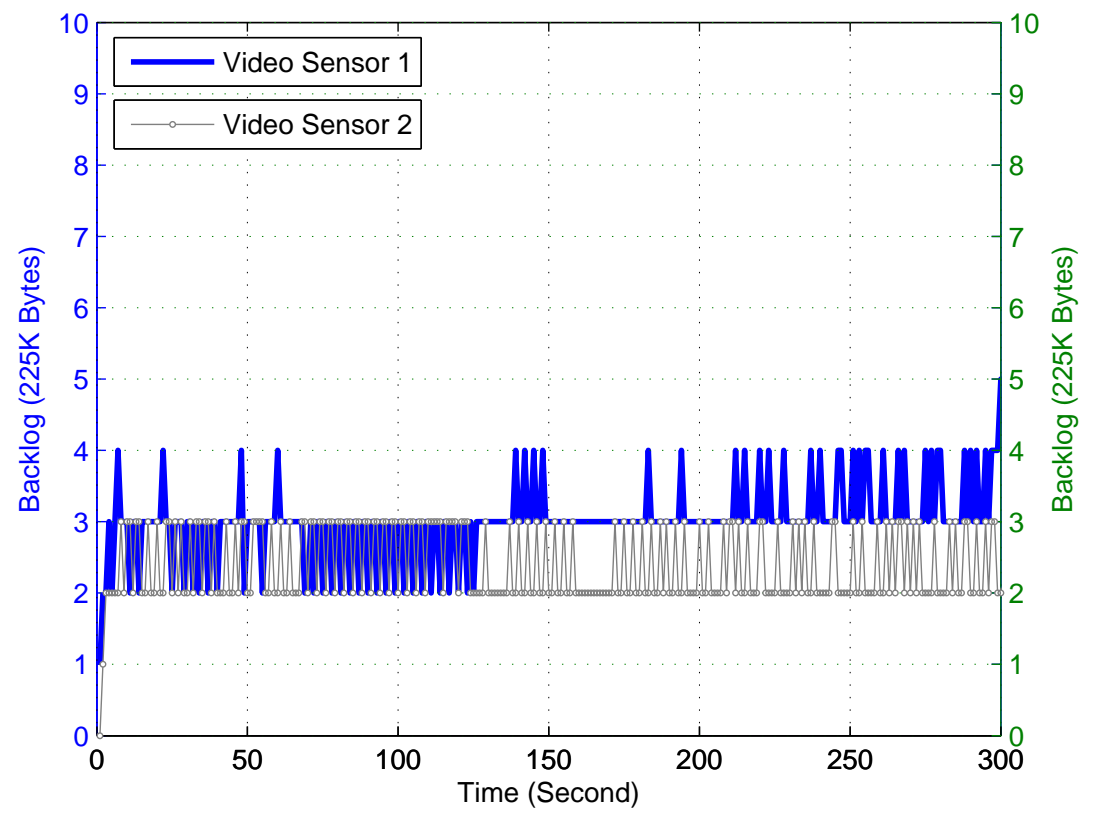

Figure V.13: Buffer and Buffer Bound in Multi-hop Scenario ( Scenario C in Fig. V.3 ) 


\section{CHAPTER VI}

\section{RELATED WORKS}

The flow based model used in this thesis is based on the work of network calculus [2]. In [2], the formal definition of the three bounds, namely Backlog Bound, Delay Bound, and Output Bound were given for general queueing systems, we derived the mathematical expression of the three bounds in video sensor network semantics, under both single- and multi-hop scenarios. The maximal clique based contention graph has been detailed discussed in [20], in [20], the clique-based discussion is used for flow-based pricing, in order to solve the corresponding primal-dual problem that maximize utility, subject to achievable bandwidth, over rate allocation within clique.

Paper [3]considered lifetime and coverage tradeoff. They proposed a protocol for node sleep scheduling that guarantees a bounded-delay sensing coverage while maximizing network lifetime.In [3], the framework is optimized for rare event detection, which is in favor of the bursty traffic in intensive events situation.

Paper [22] considered the bandwidth and scheduling issue, it present a bandwidth management framework that coordinate multiple video streams, but doesn't explicitly take energy efficiency as optimization goal.

Paper [6] considered maximized the functional lifetime, which refers to maximum number of times a certain data collection function or task can be 
carried out without any node running out of energy. Therefore it formulated and solved linear programming problem. Under the upper bound of energies and data, optimal communication strategy is derived. While our research focused on efficiently using sarcastic resource under the constraint of communication facility.

Paper [16] considered energy, coverage and bandwidth issues integrally, but did not discussed in detail, no analytical bounds or approaches have been provided. Other effort to reduce the power consumption in wireless sensor network either introduce extra hardware or middleware [7], e.g. hierarchical infrastructure or multi-tier sensor system [14], or require assistance of other information. e.g. extra synchronization [18] [21] or messaging between peers beside necessary coordination. Other related works on the energy efficient sensor networks, include taking advantage of the spacial redundancy in sensor node deployment[15]. 


\section{CHAPTER VII}

\section{CONCLUSION}

In this thesis, we investigated the power management problem in the context of sleep/awake scheduling. We adopt a network calculus approach, through which we derived the service curve, buffer and delay bound under single-hop and multi-hop scenarios. Our analysis has been validated through experiments conducted on a video sensor network testbed. Further optimization on the energy consumption could be done by adjusting the scheduling algorithm subject to the service, buffer, and delay constraints. For example, improve the energy model to relate scheduling-related variables to the three bounds and solve the energy consumption minimization problem by formatting it as linear programming problem.Our bounded experiment results validate the presentness of the optimal solution.Other improvement includes formulate more realistic and complex contention model beside and compared with our current clique model, and implement proper approximation algorithm to efficiently simplify them. 


\section{BIBLIOGRAPHY}

[1] J.-Y.Le Boudec and O.Verscheure. Optimal smoothing for guaranteed service. In IEEE/ACM Trans. Networking, volume 8, pages 689-696, 2000.

[2] Jean-Yves Le Boudec and Patrick Thiran. Network Calculus:A Theory of Deterministic Queuing Systems for the Internet. Springer, 2001.

[3] Q. Cao, T. Abdelzaher, T. He, and J. Stankovic. Towards optimal sleep scheduling in sensor networks for rare-event detection. In Proc. of IPSN, 2005.

[4] Y. Cao, Y. Xue, and Y. Cui. Network-calculus-based analysis of power management in video sensor networks. In ISIS Technical Report (http://eecs.vanderbilt.edu/people/yuanxue/publication-files/vsn.pdf).

[5] Wu-Chi Feng, Ed Kaiser, Wu Chang Feng, and Mikael Le Baillif. Panoptes: scalable low-power video sensor networking technologies. ACM Trans. Multimedia Comput. Commun. Appl., 1(2):151-167, 2005.

[6] Arvind Giridhar and P.R. Kumar. Maximizing the functional lifetime of sensor networks. In Proc. of IPSN, 2005.

[7] Xiaohui Gu and Klara Nahrstedt. An event-driven, user-centric, qosaware middleware framework for ubiquitous multimedia applications. In M3W: Proceedings of the 2001 international workshop on Multimedia middleware, 2001.

[8] Chao Gui. Virtual patrol: A new power conservation design for surveillance using sensor networks. In Proc. of IPSN, 2005.

[9] Chao Gui and Prasant Mohapatra. Power conservation and quality of surveillance in target tracking sensor networks. In ACM MOBICOM, 2004.

[10] Bret Hull, Kyle Jamieson, and Hari Balakrishnan. Poster abstract: bandwidth management in wireless sensor networks. In Proc. of SenSys, 2003. 
[11] Bret Hull, Kyle Jamieson, and Hari Balakrishnan. Mitigating congestion in wireless sensor networks. In Proc. of SenSys, 2004.

[12] Iranli, Fatemi A., and M. H., Pedram. Lifetime-aware intrusion detection under safeguarding constraints. In Proc. of IPSN, 2005.

[13] M. Krunz and S. Tripathi. Impact of video scheduling on bandwidth allocation for multiplexed mpeg streams. In Multimedia Sys. J., volume 5, pages 347-357, 1997.

[14] Purushottam Kulkarni, Deepak Ganesan, Prashant Shenoy, and Qifeng Lu. Senseye: a multi-tier camera sensor network. In Proc. of MULTIMEDIA, 2005.

[15] M. Pedram M.Maleki. Qom and lifetime-constrained random deployment of sensor networks for minimum energy consumption. In Proc. of IPSN, 2005.

[16] Curt Schurgers Sachin Adlakha, Saurabh Ganeriwal and Mani B. Srivastava. Poster abstract: density, accuracy, delay and lifetime tradeoffs in wireless sensor networks a multidimensional design perspective. In Proc. of SenSys, 2003.

[17] Bhaskar Krishnamachari Sundeep Pattem, Sameera Poduri. Energyquality tradeoffs for target tracking in wireless sensor networks. In Proc. of IPSN, 2003.

[18] Yu-Chee Tseng, Chih-Shun Hsu, and Ten-Yueng Hsieh. Power-saving protocols for ieee 802.11-based multi-hop ad hoc networks. Comput. Networks, 43(3):317-337, 2003.

[19] Chieh-Yih Wan, Shane B. Eisenman, and Andrew T. Campbell. Coda: congestion detection and avoidance in sensor networks. In Proc. of SenSys, 2003.

[20] Yuan Xue, Baochun Li, and Klara Nahrstedt. Optimal resource allocation in wireless ad hoc networks: A price-based approach. In IEEE Trans. Mobile Computing, volume 5, pages 347-364, 2006. 
[21] Ness B. Shroff Yan Wu, Sonia Fahmy. Optimal sleep/wake scheduling for time-synchronized sensor networks with qos guarantees. In $I W Q o S$ '06, 2006.

[22] K Nahrstedt Z Yang. A bandwidth management framework for wireless camera array. In Network and Operating System Support for Digital Audio and Video(NOSSDAV), 2005.

[23] R. Zheng and R. Kravets. On-demand power management for ad hoc networks. In Proc. of IEEE INFOCOM, 2003. 